\title{
Contribución a la Eficiencia Energética en la Empresa SEATEC
}

\author{
Raúl Alejandro Martínez Sánchez (Ing.), \\ Especialista de proyectos, Empresa SEATEC S.A, Santa Elena, Ecuador \\ C. Idalberto Macías Socarrás (Dr.), \\ Universidad Estatal Península de Santa Elena (UPSE), \\ Facultad de Ciencias Agropecuarias, Santa Elena, Ecuador \\ Antonio Luis Barrera Amat (M.Sc.), \\ Escuela Superior Politécnica del Chimborazo, Sede Amazónica del Norte, \\ ESPOCH, Ecuador
}

Doi: 10.19044/esj.2019.v15n15p109 URL:http://dx.doi.org/10.19044/esj.2019.v15n15p109

\section{Resumen}

La investigación, se desarrolló en la empresa SEATEC, ubicada en las siguientes coordenadas $2^{\circ} 13{ }^{\prime} 39.5^{\prime \prime} \mathrm{S}$ y $80^{\circ} 54^{\prime} 38.1^{\prime \prime} \mathrm{W}$ en el cantón La Libertad, provincia Santa Elena, Ecuador. La entidad se encuentra dedicada a la ejecución de proyectos para el sector acuícola y a la comercialización de productos de ferretería tanto para el sector naval como industrial. El objetivo perseguido fue la elaboración de un plan de mejoras que contribuyera a elevar la eficiencia energética, utilizando instrumentos de medición de parámetros eléctricos para determinar la situación de la energía en la organización; y la norma ISO 50001 para evaluar el comportamiento de la política energética empresarial. El uso de estas herramientas permitió conocer que los motores eléctricos instalados eran los causantes de cerca del 60\% del consumo eléctrico total de la empresa y que la eficiencia real calculada con la que se encontraban trabajando estos equipos como promedio era del $85.9 \%$, valor muy debajo del dato de placa del fabricante $(90.2 \%)$. Además, se aplicaron una serie de encuestas y capacitaciones basadas en la norma ISO 50001 que permitieron determinar oportunidades de mejora en los procesos de producción, esto repercutió de manera significativa en la mejora de los índices de eficiencia energética.

Palabras Clave: Eficiencia energética, motores, herramientas, norma ISO 


\title{
Contribution to Energy Efficiency in the Company SEATEC
}

\author{
Raúl Alejandro Martínez Sánchez (Ing.), \\ Especialista de proyectos, Empresa SEATEC S.A, Santa Elena, Ecuador \\ C. Idalberto Macías Socarrás (Dr.), \\ Universidad Estatal Península de Santa Elena (UPSE), \\ Facultad de Ciencias Agropecuarias, Santa Elena, Ecuador \\ Antonio Luis Barrera Amat (M.Sc.), \\ Escuela Superior Politécnica del Chimborazo, Sede Amazónica del Norte, \\ ESPOCH, Ecuador
}

\begin{abstract}
This study was carried out at SEATEC, located at the following coordinates $2^{\circ} 13^{\prime} 39.5$ "S and 80 $54^{\prime} 38.1^{\prime \prime} \mathrm{W}$ in the canton of La Libertad, province of Santa Elena, Ecuador. The entity is dedicated to the execution of projects for the aquaculture sector and the commercialization of hardware products for both the naval and industrial sectors. This paper focuses on elaborating the improvement plan that would contribute to elevate the energetic efficiency, using instruments of measurement of electrical parameters, to determine the situation of the energy in the organization. It highlights how ISO 50001 standard is used to evaluate the behavior of the company's energy policy. The use of these tools have helped us to ascertain that the electric motors installed were the cause of about $60 \%$ of the company's total electricity consumption and that the calculated real efficiency with which these teams were working as an average was $85.9 \%$. This is very below the manufacturer's plate data (90.2\%). In addition, a series of surveys and trainings based on the ISO 50001 standard were applied, which allowed us to determine opportunities for improvement in the production processes. Hence, this had a significant impact on the improvement of the energy efficiency indexes.
\end{abstract}

Keywords: Energy efficiency, motors, tools, ISO standard

\section{Introduction}

Durante el desarrollo de la humanidad el consumo de energía ha tenido una tendencia creciente, aumentando bruscamente a partir de la Revolución Industrial. El esquema energético desde comienzos del siglo XX 
ha estado basado fundamentalmente en combustibles fósiles, principalmente petróleo y carbón, los cuales suministran hoy en día más del 70\% de la energía consumida a nivel mundial. En los inicios del desarrollo industrial y hasta décadas pasadas el consumo energético no constituía un problema para la humanidad, pues existía una abundancia relativa de combustibles fósiles y no era tan evidente la afectación causado sobre el medio ambiente, a partir de la década de los 70 los impactos ambientales alcanzaron tal magnitud que el hombre comenzó a preocuparse por estos efectos (Borroto \& Monteagudo, 2011).

El mantenimiento del sistema energético actual durante un plazo de tiempo de una o dos generaciones a partir del año 2000 es simplemente insostenible porque está agotando las reservas de combustibles, contribuye al aumento del efecto invernadero, a la acidificación del agua, a la deforestación y origina riesgos para la paz mundial. (Nordelo \& Padrón, 2002; Nordelo, 2006).

A partir del año 2000 se han podido observar grandes cambios en el sistema energético mundial: el rápido despliegue de las energías renovables, la disminución de los costos de las tecnologías de energía limpia y la creciente electrificación mundial.

A escala mundial, la energía consumida por el hombre proviene en más de un $80 \%$ de combustibles fósiles (carbón, petróleo y gas natural) cuya utilización masiva conduce al agotamiento de sus reservas y supone una amenaza real al medio ambiente, que se manifiesta, principalmente, a través del calentamiento global de la Tierra y de la acidificación del agua (Nordelo \& Monteagudo, 2007).

Es por esto que los mayores esfuerzos se encuentran dirigidos al desarrollo de sistemas energéticos basados en alternativas de menor costo, con énfasis en fuentes renovables como energía solar, hidroeléctrica, eólica, geotérmica y haciendo especial hincapié en la eficiencia energética.

De acuerdo al informe presentado por la agencia internacional de energía (IEA), las necesidades globales de energía se estiman que aumentan más lentamente que en las décadas posteriores, pero aún se expanden en un $30 \%$ en un período comprendido entre el año 2015 y hasta el 2040. Esto es aproximadamente el equivalente de agregar a China e India a la demanda mundial actual.

Las perspectivas de la situación energética actual no son optimistas. No se debe olvidar que todos los países realizan continuos esfuerzos en un intento de mejorar su situación socio-económica. De aquí se desprende que si la economía mundial progresa, la demanda de energía tiende a crecer igualmente, por lo que es vital lograr un uso eficiente lo que se traduce en reducir los consumos (Nordelo, 2002; Nordelo, 2006). 
La aparición en los últimos años de economías emergentes que demandan grandes cantidades de energía, como China, India y Brasil, agravan aún más el panorama energético mundial. Estudiosos del tema prevén que para el 2050 se habrán agotado las fuentes tradicionales de energía. En todo el mundo existe la preocupación sobre el porvenir energético mundial, sin embargo, hay una gran contradicción entre los resultados de los estudios sobre la energía y la política de muchos países capitalistas con sociedades de consumo con hábitos sumamente derrochadores a los que no se pone coto con una política coherente y racional (Nordelo, 2009; Carretero, 2012).

Latinoamérica está expuesta a una crisis energética en un escenario en que cada vez es más difícil aprobar nuevos proyectos de generación debido a la presión que ejercen grupos ambientalistas y donde varios países tienden a alterar las reglas del juego y adoptar decisiones que generan inseguridad jurídica para las inversiones.

Los países de esta región del planeta no pueden apostar exclusivamente a un tipo de generación energética ni tampoco depender del abastecimiento de un único proveedor. La estrategia debe ser una diversificación de la matriz que permita tener energía segura, limpia y barata, sobre la base de estimular soluciones de mercado con regulaciones transparentes, técnicas y eficientes (OLADE, 2016).

Otro componente de la realidad de América Latina es que el crecimiento económico plantea una mayor demanda de recursos energéticos, lo cual incrementa las vulnerabilidades de algunos países y deja al descubierto las falencias de otros. Hay pues un riesgo que puede expresarse no sólo en la escasez de recursos energéticos, sino también en el agotamiento de las reservas existentes, en la reducción de la producción o incluso en la disminución de la calidad de la misma cuando nos referimos a los combustibles fósiles, pero también se presenta en la generación de energía, para la cual cada día se imponen más barreras, lo que acrecienta los riesgos asociados independiente de sus fuentes (OLADE, 2016).

El diagnóstico se cierra considerando que mientras aumenta la demanda y se consolida el déficit en exploración, explotación, infraestructura para su producción, transporte y generación de energía, todo lo cual requiere cuantiosas inversiones, varios estados latinoamericanos responden a esta situación con medidas gubernamentales que generan inseguridad jurídica en el ambiente de negocios.

Ecuador es un país en desarrollo que con la explotación petrolera empezó la modernización de su economía, moviéndose hacia un mayor crecimiento económico y consumo energético. Como resultado de ello, ha tenido una demanda de energía creciente y una modernización de su matriz energética que constituyó a los combustibles fósiles en la fuente principal de energía para el país y a la hidroelectricidad en la principal fuente de 
electricidad. Así, en 2008, el petróleo representaba el $84 \%$ de la matriz energética en el país y la hidroelectricidad el 59\% de la matriz eléctrica, con un $38 \%$ de electricidad generada en centrales térmicas de combustibles fósiles, mientras que otras fuentes de energía renovable como solar, eólica y geotérmica no constituían ni el 1\% (CONELEC, 2010; OLADE, 2016).

Un elemento de política clave y que ha influido fuertemente en la matriz energética en Ecuador son los subsidios a derivados de petróleo. Puede argumentarse que por la presencia de estos subsidios el país muestra un crecimiento mayor que los países andinos en su demanda de energía, en especial en el sector transporte. Este crecimiento en la energía no se debe en esencia a un uso más productivo y eficiente de la energía en el desarrollo de actividades de alto valor agregado, sino a un consumo mayor en energía para transporte y para el sector residencial.

La Agencia Internacional de la Energía (IEA) publicó en el 2016 el informe Energy Efficiency Market Report, este documento analiza el avance obtenido en el uso de mecanismos de eficiencia energética. En el mismo se plasma que la intensidad energética global se ha visto reducida en un 1,8\% durante 2015, superando la mejora experimentada en $2014(1,5 \%)$ y el promedio de la última década $(0,6 \%)$. Además, este avance es de especial relevancia al darse en un contexto de disminución de precios de los recursos energéticos, con el precio del petróleo crudo cayendo en un 60\% desde 2014, que suele incentivar su uso.

El aumento de la eficiencia energética fue más acusado en países emergentes y en vías de desarrollo, como India e Indonesia, en comparación con otros países más industrializados. Cabe destacar el caso de China, cuya eficiencia energética se elevó un 5,6\% en 2015. El progreso de este país tiene un notable impacto a nivel global. Si no se considerara el incremento de eficiencia chino, el porcentaje de mejora descendería, hasta el 1,4\% (Pérez, 2016).

Las medidas de eficiencia energética están posibilitando la reducción de la intensidad y la demanda energética. Así, entre el año 2000 y el 2015, los niveles de eficiencia energética en los países miembros de la IEA se incrementaron en un $14 \%$, de media. Como consecuencia, se produjo un ahorro de 450 millones de toneladas equivalentes de petróleo en 2015, suficiente para abastecer a Japón durante un año entero (Pérez, 2016).

Sin embargo, esta positiva evolución de los últimos años sigue sin ser suficiente y se requieren esfuerzos adicionales en mejorar la intensidad y la eficiencia energéticas: la IEA estima que habría que reducir urgentemente la intensidad energética hasta un 2,6\% anual para conseguir los objetivos medioambientales globales (Pérez, 2016).

Debido a los esfuerzos de organismos internacionales tanto gubernamentales como no gubernamentales se han suscrito acuerdos para 
realizar una explotación racional de los recursos energéticos del planeta y regular la emisión de gases de efecto invernadero a la atmósfera. Muchos expertos coinciden en que una de las formas para hacer un mejor uso de los recursos energéticos es la implementación de políticas y sistemas que mejoren la eficiencia energética tanto en el sector industrial como residencial. Mejorando los índices de consumo se disminuyen las emisiones de $\mathrm{CO}_{2}$ y además se contribuye al ahorro de los combustibles fósiles y otras fuentes no renovables utilizadas para la generación de energía.

La eficiencia energética es un concepto que ha venido evolucionando durante las últimas décadas. Su desarrollo ha venido a la par con el aumento de la demanda energética a nivel mundial. A medida que los combustibles fósiles han comenzado a escasear se ha hecho evidente la necesidad de crear sistemas y procedimientos que sean capaces de hacer un uso más eficiente de la energía. Gestionar la energía de manera eficiente se ha convertido en una prioridad tanto para el sector industrial como residencial.

Por todos estos beneficios las empresas deberían implementar políticas o sistemas de gestión de la energía, con el objetivo de optimizar el uso de los portadores energéticos, tan crítico en la mayoría de los procesos industriales a corto, mediano y largo plazo. Esta norma constituye el instrumento más recomendable para las organizaciones que quieren realizar una gestión eficiente de la energía.

La empresa SEATEC, no posee un sistema para la gestión energética que garantice la máxima eficiencia energética, por lo que la presente investigación tiene como objetivo elaborar un material de carácter científicotécnico mediante la implementación de la normas ISO 50001 y la aplicación de métodos técnico económicos que permitan dotar a la empresa SEATEC, de una herramienta efectiva que permita la implantación y desarrollo de un sistema de gestión energética confiable.

\section{Materiales y Métodos}

La investigación se desarrolló en la empresa SEATEC, la misma fue fundada en el año 1990. Su primera ubicación fue en el cantón Santa Elena; en el año 2000 fue trasladada al cantón La Libertad. La organización cuenta con instalaciones que ocupan un área de $4400 \mathrm{~m}^{2}$. 


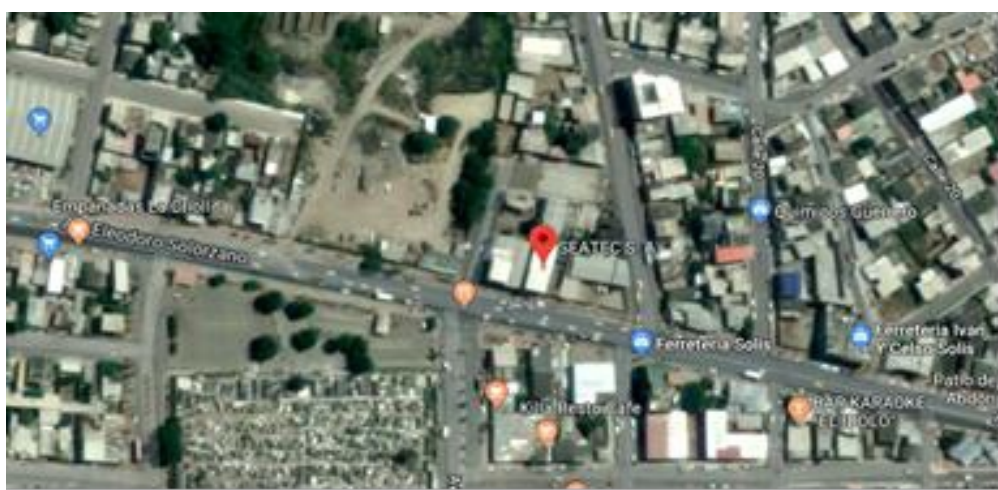

Figura 1. Ubicación de la empresa SEATEC

SEATEC tiene como misión principal proporcionar servicios y materiales de la más alta calidad para satisfacer las necesidades de la industria acuícola, naval y de la construcción y cuenta con dos líneas principales de trabajo: la comercialización al por mayor y menor de artículos para la construcción y ferretería; tanto para el sector industrial y naval como la venta de productos para el sector acuícola, fundamentalmente para la rama camaronera.

\section{Estructura organizativa empresarial}

La organización se encuentra dividida en 8 departamentos, gerencia: se encuentra dividido en dos áreas, gerencia administrativa-financiera y gerencia de innovación y desarrollo. Ventas: Se encuentra dividida en dos áreas, ventas Web y ventas VIP. Los restantes departamentos son, logística, almacén, recursos humanos, proyecto, laboratorio y contabilidad-finanzas.

\section{Recursos y materiales utilizados}

Durante el estudio del comportamiento energético de la empresa se registraron las principales magnitudes eléctricas de los equipos y sistemas de mayor consumo, para ello se emplearon los registradores de energía (PM-800) que se encuentran instalados en los diferentes paneles de control de la institución, estos se encuentran conectados en red al sistema SCADA PowerStudio del fabricante Circutor. Además, se utilizó el analizador de red Fluke (ver Figura 2) y se hicieron mediciones puntuales a diferentes equipos con la pinza amperimétrica, con el fin de mejorar la precisión de los datos obtenidos. 


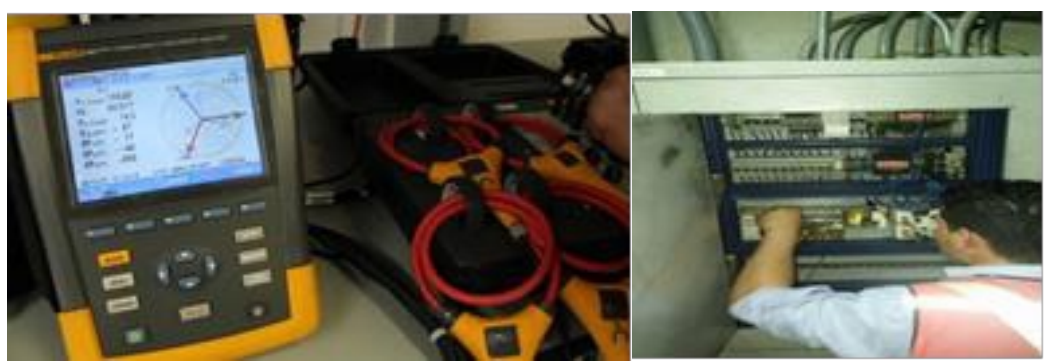

Figura 2. Etapa de medición con analizador de red Fluke

\section{Analizador de red Schneider Electric PM-800}

Con este equipo se midieron parámetros eléctricos como el voltaje, corriente, potencia, factor de potencia, frecuencia, armónicos, estos fueron instalados en la pizarra general de distribución presente en cada área (Ferretería, Taller y Laboratorio) de la empresa. Para el registro de las mediciones se instalaron 3 equipos en toda la organización, estos se encontraban conectados a través de un bus Modbus RS-485 lo cual permitió la recopilación de los datos de los parámetros en tiempo real y la exportación de estos a través de un convertidor RS484-USB al software PowerStudio.

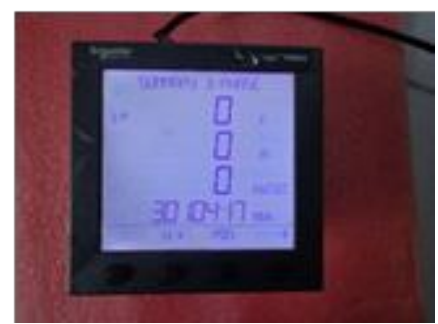

Figura 3. Analizador de Red PM-800

\section{Analizador de red Fluke 435 II}

Este equipo es un analizador de red portátil, el dispositivo ofrece un conjunto potente y completo de medidas para comprobar sistemas de distribución eléctrica. Con esta herramienta se realizaron las mediciones de las diferentes variables, tales como tensión, amperaje, potencia, frecuencia, factor de potencia; que se consumen en los centros y subcentros de carga pertenecientes a la empresa. La toma de valores se realizó con diferentes intervalos (1 min, $10 \mathrm{~min}, 30 \mathrm{~min}, 60 \mathrm{~min}$ ). Los datos obtenidos por el equipo fueron exportados mediante un software a través de un puerto de comunicación presente en el dispositivo hacia la base de datos, desde donde se analizaron el resultado de las mediciones y se conformó una visual clara y objetiva de la situación energética de la empresa. 


\section{Software PowerStudio de Circutor}

En la empresa se encuentra instalado el software de gestión de la Eficiencia Energética de la empresa Circutor llamado PowerStudio, este es un sistema de control y adquisición de datos (SCADA), que engloba todas las herramientas necesarias para gestionar los equipos de control energético. El uso del software permitió leer en tiempo real los valores instantáneos de todos los analizadores de red conectados a un bus Modbus RS485, de esta forma se fue analizando en tiempo real el comportamiento energético de los diferentes sistemas eléctricos instalados en la organización. Además los datos obtenidos fueron almacenados en una base de datos, con estos se realizaron tablas y gráficos que permitieron confeccionar análisis exhaustivos para determinar los horarios de mayor demanda eléctrica, el tiempo de uso de cada equipo, el consumo eléctrico de cada área. Además, con toda la información recopilada por el software se pudo ejemplificar de una manera sencilla y directa la situación energética de la empresa.

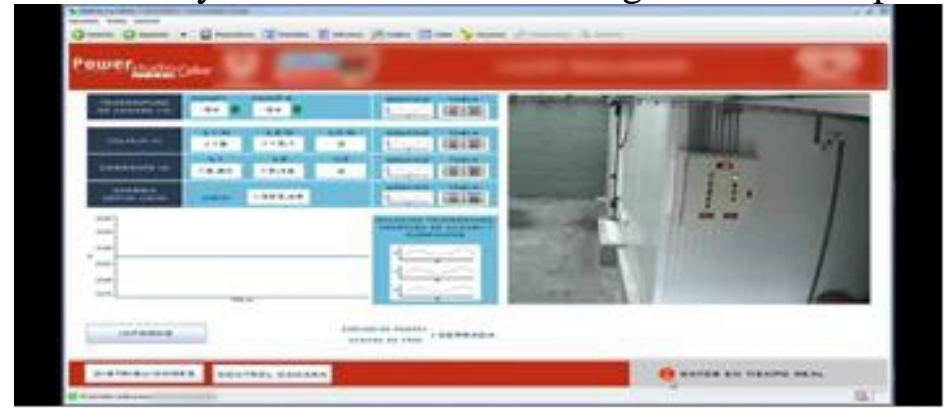

Figura 4. Imagen del Software PowerStudio de Circutor instalado en la empresa

\section{Pinza amperimétrica}

Este instrumento fue usado para medir los consumos de corriente de los equipos instalados en campo puesto que su versatilidad y precisión al momento de realizar las mediciones permitió obtener lecturas precisas de los amperajes consumidos por fases en los motores, bombas, equipos de climatización y otros equipos instalados en la empresa.

\section{Auditoría energética}

Este análisis fue realizado para trazar el flujo de energía dentro de la empresa, el objetivo principal era la identificación de oportunidades para reducir la cantidad de energía que se consume en las actividades administrativas y productivas de la organización. Además, hacer un levantamiento de todos los sistemas eléctricos instalados y determinar el estado en el que se encontraban. 


\section{Técnicas científicas de investigación}

Como parte del proceso investigativo se sostuvo encuentros con la dirección de la empresa, con el objetivo de determinar los flujos de energía de la instalación. Se indagó sobre la política energética existente en la empresa y cuáles eran las acciones que se encontraban delimitadas en la misma. También se realizó una inspección visual de las instalaciones para familiarizarse con la entidad y la operación de las diferentes áreas empresariales.

Conociendo que uno de los objetivos principales de la norma ISO 50001, es garantizar la mejora de los índices de eficiencia energética, se realizó una encuesta al personal de la empresa, la cual estaba debidamente estructurada y ajustada a la información que se requiere en la presente investigación.

\section{Encuesta acorde la Norma ISO 50001}

Se procedió a formular un cuestionario de preguntas basado en las directrices descritas en la norma ISO 50001 que permitió medir diferentes variables. Además, posibilitó la observación de los hechos a través de la valoración que se hizo a las personas encuestadas. Se realizó la verificación del cuestionario para revisar el orden de las preguntas y su clara redacción de manera que pudiera ser comprendido por el personal que conforma la muestra.

\section{Población y Muestras}

La población que conforma la empresa SEATEC es de 66 personas. Para lograr una muestra significativa se realizó el estudio con el 70\% de la población. El tipo de muestreo utilizado fue el muestreo probabilístico aleatorio simple, que permite que cada unidad que compone la población tiene la misma posibilidad de ser seleccionado. Para calcular la muestra se utilizó la siguiente ecuación:

$$
m=\frac{N}{(N-1) * K^{2}+1}
$$

Donde; $\mathrm{m}$ es el valor de la muestra y $\mathrm{N}$, la población o universo. El valor $\mathrm{K}$ representa el margen de error estimado en la investigación que fue de un 5\%. La muestra seleccionada para la aplicación de la encuesta fue de 4 directivos, 7 miembros del personal administrativo, 17 del personal técnico, 3 del personal de mantenimiento y 15 obreros. Esta fue aplicada en el mes de marzo de 2017. 


\section{Análisis y Discusión}

En las figuras 5, 6 y 7 se muestran los porcentajes de consumo por equipos en las áreas del Taller, Ferretería y Laboratorio respectivamente. Los datos mostrados vienen dados por el análisis de las fichas técnicas, datos de placa, y las mediciones realizadas específicamente a cada equipo con el analizador de red Fluke y la pinza amperimétrica.

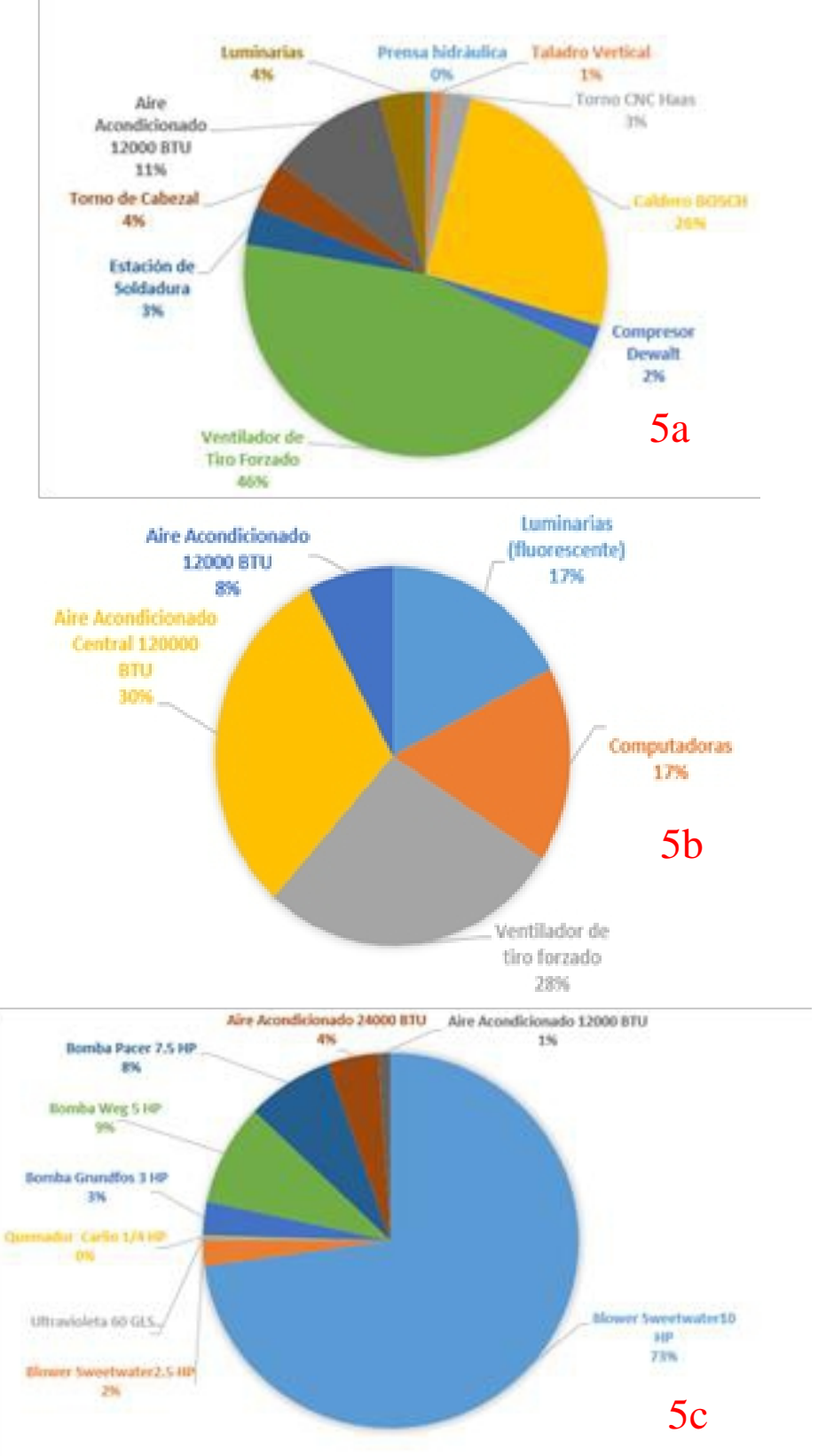

Figura 5. Distribución del consumo de energía por sistema de equipos en el taller, ferretería y laboratorio 
Barreda (2012) plantea que los motores eléctricos representan el 60\% del consumo energético mundial. Los resultados arrojados por las mediciones corroboran lo anterior. Los motores instalados para lograr la adecuada ventilación de las diferentes áreas del taller representan el 46\% del consumo total, esto significa que aumentando la eficiencia de estos equipos es posible lograr un ahorro energético considerable en la facturación eléctrica mensual. De acuerdo al estudio realizado por la Asociación de Empresas de Eficiencia Energética (AEE, 2013) de España, se plantea que como promedio dentro de un edificio de oficinas el consumo de los sistemas de ventilación y climatización es del $47 \%$, el del sistema de iluminación interior y exterior es del $26 \%$ y el consumos de los equipos de cómputo es del 13\%. Analizando los resultados mostrados en la Figura 5a se puede observar que existe una gran similitud entre los porcentajes indicados en el estudio y los obtenidos en las mediciones realizadas a los sistemas en la ferretería, aunque cabe señalar que en el caso de la iluminación los porcentajes difieren en gran medida, esto se debe a que en la empresa SEATEC no existen sistemas de alumbrado exterior.

En el laboratorio se puede apreciar que los motores eléctricos instalados son los causantes del consumo del $95 \%$ de toda la energía eléctrica del área. Este dato se encuentra a tono con la aseveración de la agencia nacional de energía Barreda (2012), donde se plantea que el 60\% del consumo mundial de energía es causado por el uso de motores. Aumentar la eficiencia real con la que operan los motores tendría un impacto positivo en la reducción del consumo eléctrico del área.

\section{Análisis del consumo de energía por sistemas de equipos}

De acuerdo a los resultados expuestos en la Figura 5 se procede a elaborar Figura 6 donde se puede observar cuanto representa cada área del consumo energético total de la empresa.

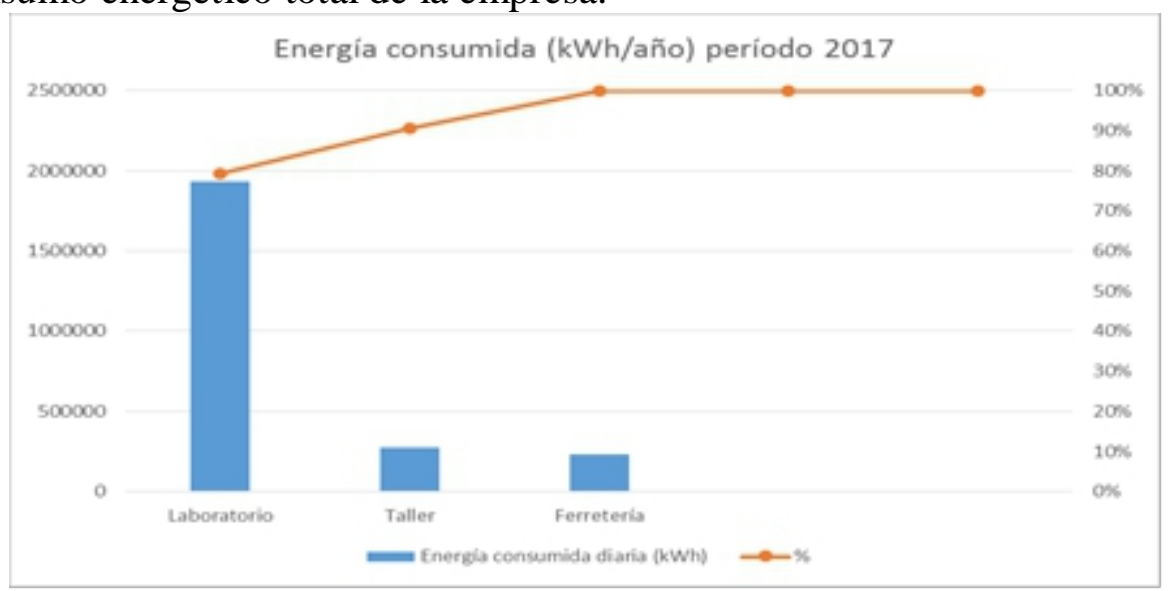

Figura 6. Energía consumida por áreas en la empresa (kWh/año) 
Con los datos obtenidos de las mediciones realizadas se concluye que el laboratorio, y por ende los motores eléctricos son los responsables del mayor porcentaje de consumo de portadores energéticos dentro de la organización. De acuerdo a la Ley de Pareto, que establece que, de forma general y para un amplio número de fenómenos, aproximadamente el $80 \%$ de las consecuencias proviene del $20 \%$ de las causas; se concluye que el laboratorio debería ser el área de la empresa donde se tomen las medidas que favorezcan al aumento de la eficiencia energética, puesto que, cualquier tipo de mejora en los índices de consumo de la misma repercutirá de manera muy favorable al consumo general de la empresa. No obstante cabe señalar que las áreas del taller y de la ferretería no están exentas de los planes de medidas a tomar derivados de este análisis.

La Figura 7 muestra cuales fueron los sistemas de equipos que mayores índices de consumo tuvieron en cada área durante el transcurso del año 2017. Esto permite conocer hacia donde deben ir dirigidas las acciones preventivas, de ahorro y de inversión con el fin de lograr mejorar el desempeño energético empresarial y por ende los índices de eficiencia energética.

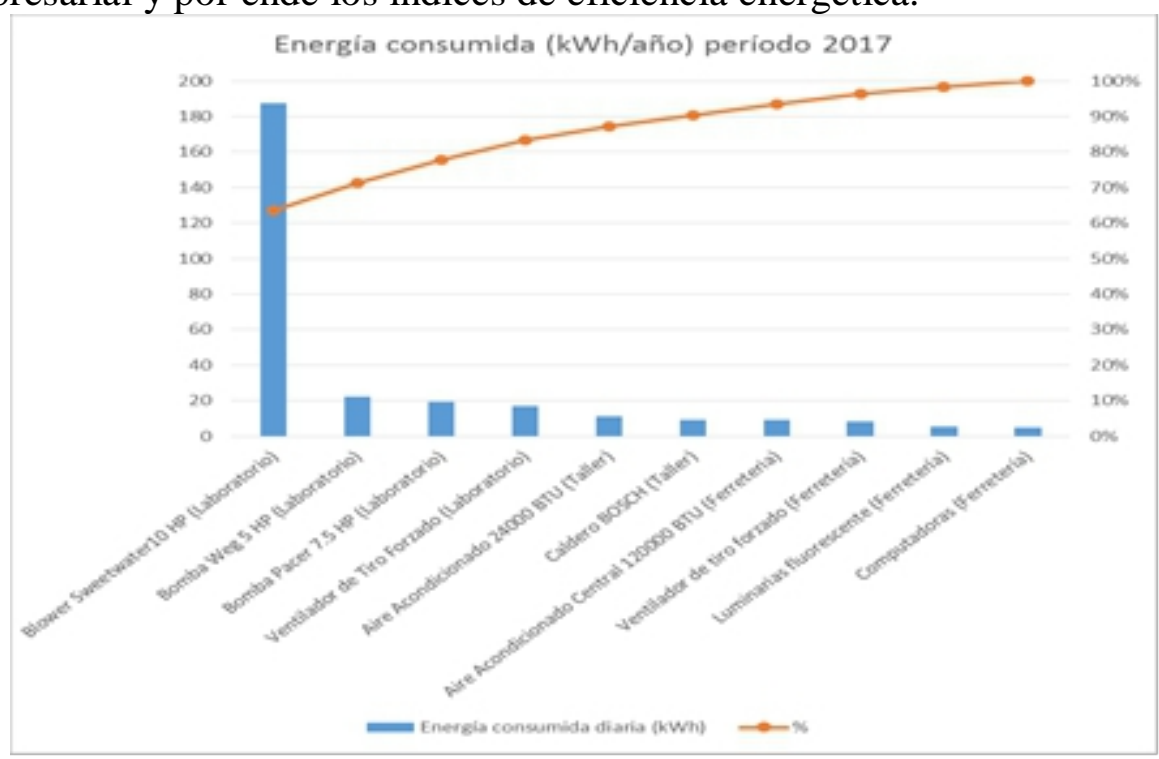

Figura 7. Energía consumida por los principales sistemas de equipos

Del análisis de la figura 7 se concluye que aproximadamente el $65 \%$ del consumo energético total de la empresa viene derivado de los motores instalados en las diferentes áreas, de acuerdo a la Ley de Pareto, si se lograra aumentar la eficiencia energética de los motores instalados en las diferentes áreas de la organización, el consumo de energía eléctrica decrecería significativamente y por ende la facturación, el caso de los sistemas de climatización y luminarias se incluyen aunque es poco significativo su consumo. 


\section{Determinación de la eficiencia real de los motores}

A lo largo de la investigación se hicieron mediciones exhaustivas para conocer fundamentalmente la eficiencia real con las que se encontraban trabajando los motores. Este proceso se hizo comprobando los datos de chapa, los resultados de las mediciones y aplicando los métodos para determinar la eficiencia.

Para el desarrollo de la investigación se utilizó el método de los datos de placa planteado por Barreda (2012) para calcular la eficiencia real del motor, este fue aplicado al motor del Blower Sweetwater de 10 HP.

Marca: WEG

Modelo: J1210B

Número de fases $(\sim): 3$

Potencia nominal (Pn): $7.5 \mathrm{~kW}(10 \mathrm{HP})$

Tensión nominal (Un): 208-230/460 V

Corriente nominal (In): 25.8-24.6/12.3 A

Corriente de arranque (Ia): (7-9)* In

Velocidad nominal: $3500 \mathrm{r} / \mathrm{min}$

Factor de potencia nominal (fpn): 0.83

Eficiencia Nominal (Nnon): 90.2 (\%)

Frecuencia: $60 \mathrm{~Hz}$

Temperatura ambiente máxima: $40{ }^{\circ} \mathrm{C}$

Factor de servicio (FS): 1.25

Potencia de entrada $\left(P_{\text {ent }}\right): P_{\text {ent }}=\sqrt{3} U_{\text {prom }} I_{\text {prom }} \operatorname{Cos} \boldsymbol{\varphi}$

Tensión entre fases (V):

- $\mathrm{U} 1-2=221.3$

- $\mathrm{U} 1-3=217.4$

- $\mathrm{U} 2-3=219.7$

$U_{\text {prom }}=219.46$

Corriente por fase (A):

- $\mathrm{I} 1=24.9$

- $\mathrm{I} 2=25.5$

- $\mathrm{I} 3=25.2$

$I_{\text {prom }}=25.2$

Factor de potencia por fase $(\%)$ :

- $\mathrm{fp} 1=78$

- $\mathrm{fp} 2=75$

- $\mathrm{fp} 3=77$ 
fp prom=76.6

Sustituyendo en la ecuación de la Potencia de entrada $\left(P_{\text {ent }}\right)$ :

$$
\begin{aligned}
& P_{\text {ent }}=\sqrt{3} U_{\text {prom }} I_{\text {prom }} \operatorname{Cos} \varphi \\
& P_{\text {ent }}=\sqrt{3} * 219.46 * 25.2 * 0.766 \\
& P_{\text {ent }}=7.3 \mathrm{~kW}
\end{aligned}
$$

Con los valores obtenidos procedemos a calcular el factor de carga:

$$
\begin{aligned}
& F_{\text {carg }}=\frac{P_{\text {ent }}}{P_{\text {nom }}} N_{\text {nom }} \\
& F_{\text {carg }}=\frac{7.3}{7.5} * 0.902 \\
& F_{\text {carg }}=0.878
\end{aligned}
$$

Ajuste por variación de tensión:

$$
\begin{aligned}
\Delta U & =\left[\left(\frac{U_{\text {prmed }}}{U_{\text {nom }}}\right)-1\right] 100(\%) \\
\Delta U & =\left[\left(\frac{219.46}{230}\right)-1\right] 100(\%) \\
\Delta U & =-4.58 \%
\end{aligned}
$$

Ajuste por desbalance de las tensiones:

$$
\begin{aligned}
& D_{\text {ifmax }}=U_{\min }-U_{\text {prom }} \\
& D_{\text {ifmax }}=217.4-219.46 \\
& D_{\text {ifmax }}=2.06
\end{aligned}
$$

Desbalance de tensiones:

$$
U_{\text {des }}=\frac{2.06}{219.46}=0.94 \%
$$

Eficiencia del motor en condiciones reales de operación:

$$
\begin{aligned}
& \eta_{\text {ajust }}=\left[\left(\eta_{f c}+\Delta U\right) U_{\text {des }}\right] \\
& \eta_{f c}: \text { evaluada solo a partir de la carga actual } \\
& \Delta U: \text { Ajuste de tensión } \\
& U_{\text {des }}: \text { Ajuste de desbalance de tensiones } \\
& \eta_{\text {ajust }}=[(90.2-4.58) * 0.99] \\
& \eta_{\text {ajust }}=84.76 \%
\end{aligned}
$$

Con la metodología utilizada para calcular la eficiencia se obtiene como resultado que el motor se encuentra trabajando con una eficiencia real del $84.76 \%$. La placa muestra que la eficiencia con la que fue diseñado es del $90.2 \%$, esto indica que los motores instalados se encuentran trabajando por debajo de los índices de eficiencia que muestran en su placa de montaje, es 
una información relevante porque significa que la potencia entrega por el motor en el eje es menor a la nominal con la que fue diseñado. Pueden existir muchos factores para obtener estos resultados, entre los que se pueden citar: deficientes intervalos de mantenimiento, obsolescencia, sobrecarga, rodamientos defectuosos.

\section{Evaluación de los resultados de las encuesta}

Al aplicar la encuesta en marzo de 2017, los resultados obtenidos demostraron que los trabajadores en general no conocen la existencia de la política energética en la empresa, ni las prácticas laborales adecuadas para mejorar los índices de eficiencia energética de la organización. Se comprobó un desconocimiento generalizado en identificar los portadores energéticos y la importancia de un uso racional de los mismos. Los trabajadores no se encontraban familiarizados con los conceptos de política energética, desempeño energético y eficiencia energética; todo esto impide mejorar los índices de consumo y la toma de decisiones acertadas en los diferentes puestos de trabajos. Se puede agregar que todas estas razones dificultan la identificación de las áreas y sistemas más proclives a las oportunidades de ahorro y mejoras.

Como parte del seguimiento de la investigación y en aras de continuar monitoreando los procesos para detectar las falencias se procedió a aplicar la encuesta nuevamente en noviembre de 2017 a los trabajadores de la empresa. A continuación se detallan los resultados obtenidos en la segunda etapa y la comparación con los resultados obtenidos en la primera encuesta.

\section{Responsabilidad de la dirección}

En la Figura 8 aparecen representados los resultados de las encuestas de cumplimiento en la etapa de responsabilidad de la dirección, las que arrojaron como resultado que los procesos en la alta dirección no se cumplían en un $90 \%$, siendo un índice muy alto y perjudicial para la empresa, pues esta es el motor para el manejo del comportamiento de la organización.
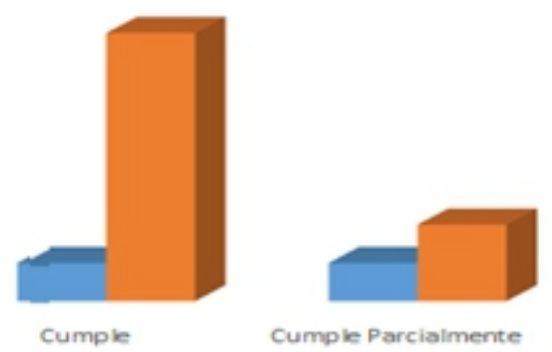

= Antes = Después

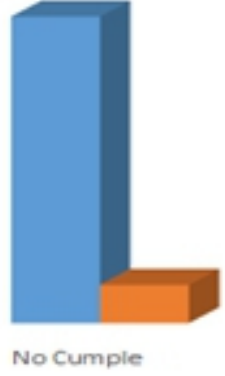

No Cumple

Figura 8. Evaluación de cumplimiento en la etapa de responsabilidad de la dirección 


\section{Verificación}

En la Figura 9 se aparecen representados los resultados de las encuestas formuladas para comprobar las etapas de verificación, destaca que el 50\% de los encuestados no cumplen con la valoración del desempeño actual de los procesos, sistemas, equipos e instalaciones, no prevén ni corrigen las no conformidades de la política energética, mientras que solo el $30 \%$ cumple, dejando un $20 \%$ en cumple parcialmente.

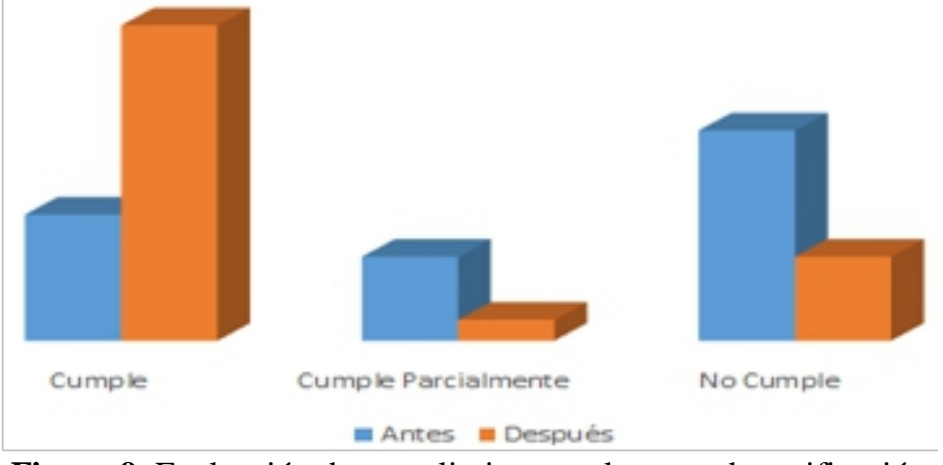

Figura 9. Evaluación de cumplimiento en la etapa de verificación

\section{Revisión por la dirección}

Analizando el cumplimiento de la etapa de revisión por parte de la dirección podemos apreciar que el $0 \%$ de los directivos cumple con los lineamientos a seguir para realizar la revisión e información a la alta dirección. El $25 \%$ cumple parcialmente y un alto porcentaje de $75 \%$ que no cumple con dicho proceso.
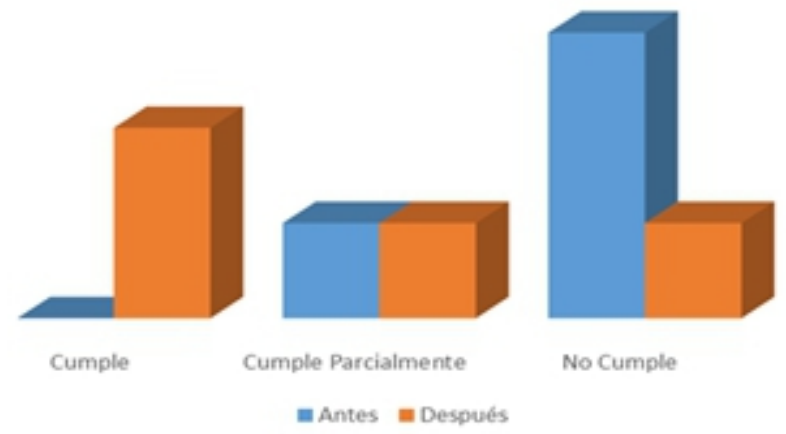

No Cumple

Figura 10. Evaluación de cumplimiento en la etapa de revisión por la dirección

De acuerdo a los resultados obtenidos se implementa que todos los parámetros que incluye la norma relacionada con este criterio se incluyen para la revisión por la dirección de la organización y se tuvieron en cuenta para la revisión por la dirección las decisiones y medidas relacionadas a la política 
energética, los objetivos estratégicos y operativos así como la provisión de recursos.

Se puede afirmar que existe una diferencia ostensible entre los resultados obtenidos en las dos encuestas. La norma ISO 50001 brindó los lineamientos necesarios para elaborar una encuesta que permitiera dibujar cual era la situación energética de la empresa SEATEC.

La primera encuesta permitió vislumbrar una situación energética no favorable en la organización. La alta dirección no tenía todas las herramientas para aplicar correctamente la política energética empresarial. Además, los trabajadores no poseían los conocimientos necesarios para aplicar criterios energéticos que favorecieran las buenas prácticas laborales, identificación de mejoras y por ende la maximización de la eficiencia energética en toda la empresa.

Con el análisis de los resultados de la segunda encuesta se observa una situación más favorable. Se puede observar como el grado de implicación de la alta dirección de la empresa es mayor. Además, los trabajadores cuentan con conocimientos y políticas que permiten una continua mejora de los procesos fabriles de la empresa y por ende la continua búsqueda de la eficiencia energética. También se percibe una mejora en la implementación adecuada de la política energética por parte de todo el personal de la organización.

\section{Consumo energético vs producción}

En la Figura 11, se aprecia como el consumo energético durante los primeros 6 meses del año sobrepasa en gran medida el volumen de producción de la empresa. Sin embargo en el resto de los meses se evidencia una tendencia a la disminución, esto se debe al resultado de las capacitaciones, talleres y charlas técnicas impartidas al personal; lo cual posibilitó identificar fugas de energía, mala manipulación de los sistemas de equipos, ciclos de mantenimiento deficientes y malas prácticas energéticas por parte de los trabajadores.

Estos resultados se encuentran a tono con lo planteado por Yanes y Gaitán (2005); Fernández, et al. (2014), donde estos autores estiman que implementar la norma ISO 50001 o seguir sus lineamientos podría influir hasta en un $50 \%$ del consumo mundial de energía. 


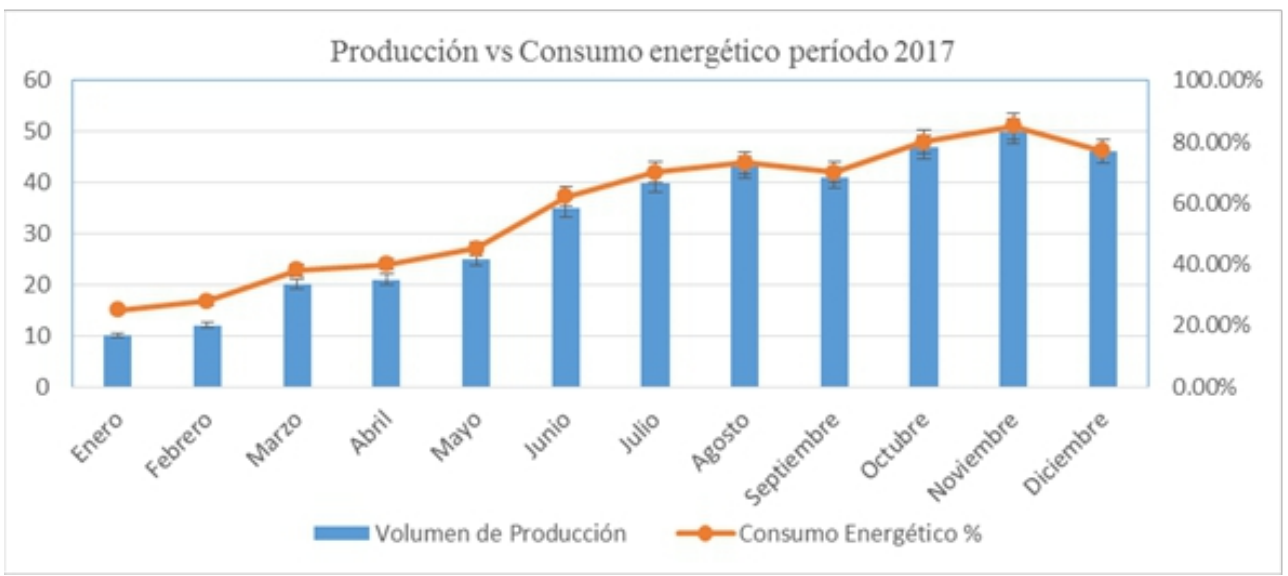

Figura 11. Consumo energético vs producción antes de implementar la norma

\section{Causas del ineficiente consumo energético}

Para delimitar cuales son las posibles causas de un ineficiente consumo energético se ha planteado un diagrama de Ishikawa (Figura 12), esta es una herramienta para el análisis de los problemas que básicamente representa la relación entre un efecto (problema) y todas las posibles causas que lo ocasionan. En el mismo se demuestra con una lluvia de ideas cuales pueden ser los posibles factores.

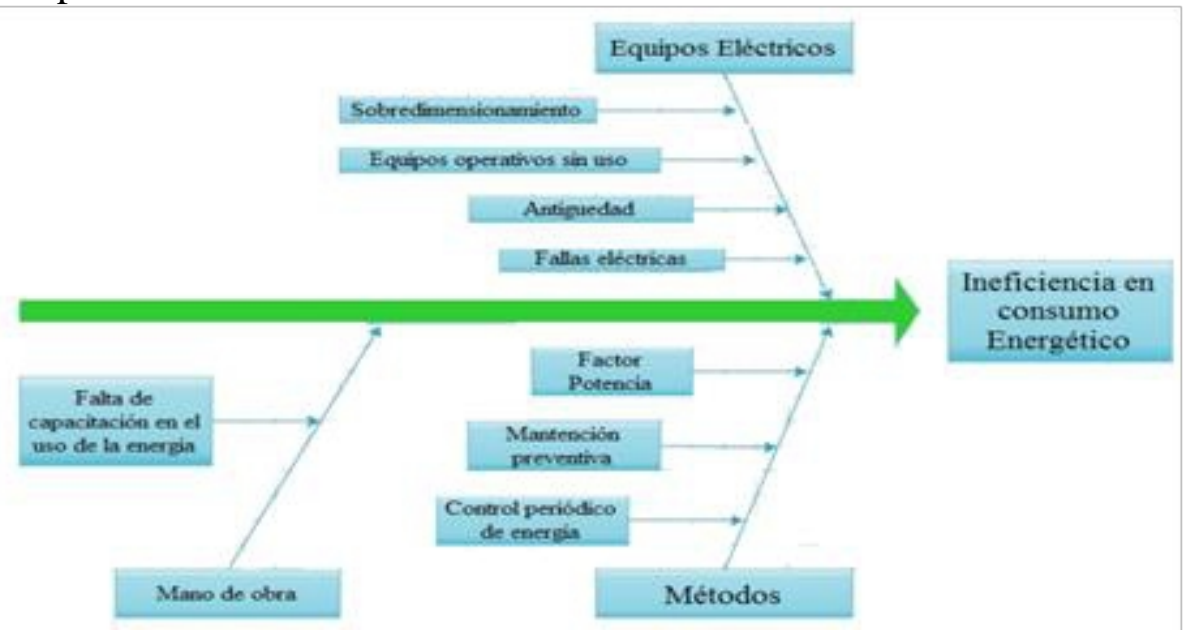

Figura 12. Diagrama de Ishikawa para la deficiencia energética

El análisis del diagrama plantea una serie de causas que pueden repercutir en mayor o menor cuantía en el correcto desempeño energético de la organización. Este tipo de herramienta permite un análisis participativo mediante grupos de mejora o grupos de análisis, que mediante técnicas como por ejemplo la lluvia de ideas, sesiones de creatividad, y otras, facilita un 
resultado óptimo en el entendimiento de las causas que originan un problema, con lo que puede ser posible la solución del mismo.

\section{Proyección de mejoras}

Partiendo del diagrama de Ishikawa se realizaron las siguientes propuestas con el fin de contrarrestar todas las deficiencias encontradas:

$\checkmark$ Disponer de un encargado de energía que tenga la capacidad de abordar y decidir temas de eficiencia energética en el centro.

$\checkmark$ Controlar las cuentas de energía y documentar cómo evolucionan consumos y gastos en el tiempo para poder detectar posibles irregularidades.

$\checkmark$ Incorporar dentro de las políticas de la empresa un estricto programa de mantenimiento de los equipos e instalaciones para asegurar su funcionamiento adecuado y evitar consumos elevados de energía.

$\checkmark$ Chequear las instalaciones con regularidad para identificar usos innecesarios de energía (por ejemplo operación de equipos sin uso/en vacío, fugas en sistemas).

$\checkmark$ Establecer procedimientos y valores de variables de operación e instruir operadores para asegurar una operación eficiente de las instalaciones.

$\checkmark$ Informar al personal respecto de la importancia de la eficiencia energética e incorporarlo a la hora de realizar acciones de eficiencia energética, ya que sus experiencias y conocimientos que resultan de su trabajo diario son valiosos, y un personal consciente y capacitado es un factor clave para alcanzar una cultura de eficiencia energética con resultados duraderos.

$\checkmark$ Propuesta de un sistema de capacitación del personal según las necesidades existentes.

$\checkmark$ Adquirir motores de alta o ultra alta eficiencia cuando se requieran nuevas aplicaciones.

$\checkmark$ Sustituir motores en mal estado o de baja eficiencia por nuevos equipos de alta o ultra alta eficiencia.

$\checkmark$ Cambiar la tecnología de iluminación (fluorescente) instalada por tecnologías más eficientes (LED) y amigables con el medio ambiente.

$\checkmark$ Utilizar pinturas de colores claros para mejorar la iluminación del entorno.

$\checkmark \quad$ Instalar nuevo sistema de climatización que use tecnología inverter.

$\checkmark$ Colocación de avisos o estampas que indiquen cómo se debe regular el sistema de climatización, siempre en $24^{\circ} \mathrm{C}$.

$\checkmark$ Realizar chequeos mensuales a los termostatos de los sistemas de climatización, con el objetivo de comprobar su correcto funcionamiento. 
$\checkmark$ Mantener puertas y ventanas cerradas de las áreas con sistemas de climatización.

$\checkmark$ Implementar un sistema automático de control de la climatización.

\section{Evaluación económica de las propuestas}

Para la valoración económica de las propuestas se tomaron en cuenta 3 sistemas de equipos: Blower sweetwater de $10 \mathrm{HP}$, sistema de climatización y luminarias.

Basado en los resultados obtenidos durante la investigación se propone el cambio de los motores de los Blowers por otros de alta o ultra alta eficiencia, puesto que de acuerdo al cálculo realizado se encuentran operando con una eficiencia del $84.76 \%$, esta se encuentra por debajo del índice de $90.2 \%$ con la que vino diseñado el equipo. Esto ayudaría considerablemente a disminuir el consumo de energía eléctrica de toda la empresa puesto que en la actualidad consumen aproximadamente el $65 \%$ de toda la demanda de la organización, resultado similar alcanzó Prashar, (2017), aunque en su caso los motores llegaron a alcanzar una eficiencia del $88 \%$.

Para realizar el análisis económico se utilizó una herramienta del fabricante WEG que se encuentra en la página web oficial de la marca. Esta permite conocer el plazo estimado para amortizar la inversión de reemplazar un motor.

En este caso el motor que se propone de reemplazo es un WEG de 10 HP con una eficiencia de $91.8 \%$. Acorde a los cálculos del software este motor va a permitir un ahorro comparado con el instalado en la actualidad de 3000 kWh/año, lo cual representan 330 USD menos en la facturación anual. El precio de este equipo oscila los 750 USD, por lo que el retorno de la inversión estaría cerca a los 2 años y 4 meses. Cabe recalcar que además con esta inversión se estarían dejando de emitir a la atmósfera $1512.34 \mathrm{kgCO}_{2} /$ año. La vida útil de este equipo está calculada para 20 años.

En caso de que todos los motores (30) fueran reemplazados, se estaría ahorrando la empresa por concepto de facturación eléctrica 9900 USD al año, además, no se consumirían $90000 \mathrm{kWh} / \mathrm{año}$ y se dejaría de emitir a la atmósfera $45370.2 \mathrm{kgCO}_{2} /$ año.

En el caso de los sistemas de climatización instalados a lo largo de toda la empresa se pudo apreciar que los equipos instalados en el $85 \%$ de los casos tienen más de 10 años de explotación. Además el gas refrigerante que usan es el R22, el cual fue descontinuada su fabricación desde el año 2010 porque contribuye al debilitamiento de la capa de ozono, los resultados coinciden con los mostrados por Jovanović et al. (2017), donde manifestaron los altos consumos de sistemas climatizados al utilizar gases refrigerantes obsoletos y equipos con desgaste debido al sobre uso. 
De acuerdo a los resultados arrojados por esta investigación se propone la modernización de los sistemas de clima por equipos que usen tecnología inverter para su funcionamiento, la cual permite ahorros de energía eléctrica de hasta un $60 \%$ y usan como gas refrigerante el R410 que es totalmente amigable con la capa de ozono y el medio ambiente.

Los sistemas de clima instalados actualmente consumen aproximadamente $222204.96 \mathrm{kWh} / \mathrm{año}$, esto representa aproximadamente 24500 USD en gastos por facturación eléctrica. En caso de modernizar los equipos y disminuir el consumo eléctrico en al menos un $30 \%$ el ahorro estaría por el orden de los 7500 USD anuales. La inversión total para lograr modernizar los equipos rondaría los 21500 USD y el tiempo de amortización se estima en 3 años.

El sistema de iluminación instalado en la empresa es totalmente obsoleto y va contra las normativas vigentes en la actualidad. Cambiar la tecnología y mejorar la calidad de la iluminación constituye una tarea prioritaria, resultados similares fueron planteados por Pacheco (2006), proponiendo medidas de ahorro y suplantación de equipos ineficientes.

Las lámparas fluorescentes son la tecnología de iluminación del pasado, estar acorde a los últimos adelantos tecnológicos se impone, sobre todo en una empresa que se encuentra empeñada en mejorar sus índices de eficiencia energética. De esta manera se propone el cambio de las luminarias instaladas por lámparas LED que van a permitir reducir el consumo energético en casi un $50 \%$, mejorar la calidad de lúmenes por metro cuadrado, además van a disminuir los costos por reposición, puesto que la vida útil de estos nuevos equipos es mayor.

Actualmente la energía consumida por las lámparas fluorescentes es de $51306.18 \mathrm{kWh} /$ año lo que representa un consumo eléctrico que asciende a 5643.76 USD. Con el cambio de tecnología el ahorro estimado esta por el orden de los 2800 USD. El monto total de la inversión sería de aproximadamente 6500 USD y el tiempo de amortización se estima en 2 años y 8 meses.

Estas propuestas se encuentran destinadas a lograr un mejor uso de los portadores energéticos, aumentan exponencialmente la eficiencia energética y mantener una actitud amigable con el medio ambiente.

\section{Conclusión}

La investigación permitió identificar los equipos con mayor impacto en el consumo total de energía de la empresa, la utilización de la norma ISO 50001 permitió a la alta dirección de la empresa detectar falencias en la política energética e identificar oportunidades de mejora en los procesos energéticos que se ejecutan dentro de la organización, finalmente se elaboró un plan de medidas encaminado a mejorar los índices de consumo energético, 
aspecto este que permitió fundamentar una propuesta energética que contribuye al aumento de la eficiencia en la empresa SEATEC.

\section{References:}

1. Barreda, A. (2012). Método práctico de obtención de potencia y eficiencias de unidades de bombeo en operación. Revista Ingeniería Hidráulica y Ambiental. VOL. XXXIII, No. 3. ISSN 1815-591X

2. Beihmanis, K. \& Rosa, M. (2016). Energy Management System Implementation in Latvia Municipalities: From Theory to Practice, Energy Procedia, vol. 95, pp. 66-70.

3. Borroto, A. (2002). Gestión energética empresarial. Cienfuegos: Universidad de Cienfuegos. ISBN: 959-257- 040-X

4. Borroto, A. \& Monteagudo, A. (2011). Gestión y Economía Energética., Universidad Carlos Rafael Rodríguez de Cienfuegos, Editorial Universo Sur, Cuba, pp. 16-49.

5. Carretero, A. (2012). Sistemas de gestión de eficiencia energética ISO 50001:2011. La contribución a la eficiencia energética de los sistemas de gestión y las auditorías energéticas. Dirección de Desarrollo Cumbre de Gestión Sostenible. Ministerio de Industria, Energía y Turismo Madrid. España, pp. 12-21 y 44-56.

6. CONELEC (2010). Estadística del Sector Eléctrico Ecuatoriano. Período 1990-2018. Quito. CD-ROM.

7. Duart, V. (2007). Eficiencia y ahorro energético: contribución del sector de las tecnologías de la información Universia Business Review, núm. 13, primer trimestre, pp. 116-127, Portal Universia S.A. España.

8. Fernández, L., Aballe, L., \& Carbonell, T. (2014). Aplicación de Gestión Total Eficiente de Energía en el Centro Internacional de Salud "La Pradera". Revista Ingeniería Energética. vol.35, n.2.

9. Jovanović, B., Filipović, J., \& Bakić, V. (2017). Energy management system implementation in Serbian manufacturing - Plan, Do, Check, Act cycle approach. Journal of Cleaner Production, vol. 162, pp.11441156.

10. Nordelo, A. \& Padrón, A. (2002). Ahorro de energía en sistemas termo-mecánicos. Centro de estudios de energía y medio ambiente. ISBN 959-257-045-0, Editorial Universidad de Cienfuegos. P 159.

11. Nordelo, A. (2006). Fundamentos de Gestión Energética y Tecnología de Gestión Total y Eficiente de la Energía. Edición Cienfuegos: Universidad de Cienfuegos.

12. Nordelo, A, \& Monteagudo, J (2007). Gestión y Economía Energética. Editorial Universo Sur. Universidad de Cienfuegos. ISBN: 959-257114. 
13. Nordelo, A. (2009). Los sistemas energéticos y sus costos ambientales., Centro de Estudios de Energía y Medio Ambiente, Universidad Carlos Rafael Rodríguez de Cienfuegos, Editorial Universidad de Cienfuegos, Cuba, pp. 19-32.

14. OLADE (2016). Panorama Energético de América Latina y el Caribe 2018. Disponible en: [http://biblioteca.olade.org/opactmp1/Documentos/old0416b.pdf], consultado: [12 de abril de 2018]

15. Pacheco, R. (2006). Sistema de gestión de energía y agua para la Universidad de Granma, Cuba. Tesis para aspirar al grado académico de Máster en Ciencias. Facultad de Ingeniería Mecánica. Universidad Carlos Rafael Rodríguez. CEEMA.

16. Pérez, D. (2016). Caso práctico de Implementación de un Sistema de Gestión de la Energía en la Industria. VII Seminario Latinoamericano y del Caribe de Eficiencia Energética, Montevideo, Uruguay, pp. 2631.

17. Prashar, A. (2017). Adopting PDCA (Plan, Do, Check, Act) cycle for energy optimization in energy intensive SMEs. Journal of Cleaner Production, vol. 145, pp. 277-293.

18. Serna, C. (2010). Gestión energética empresarial una metodología para la reducción de consumo de energía, Producción + Limpia, ISSNe 1909-0455, Vol. 5, No. 2. pp 107-126.

19. Yanes, J. \& Gaitán, O. (2005). Herramientas para la gestión empresarial. Scientia et Technica, ISSN 0122-1701, Vol. 3, No. 29, págs. 169-174. 